\title{
Refractory Follicular Lymphoma
}

National Cancer Institute

\section{Source}

National Cancer Institute. Refractory Follicular Lymphoma. NCI Thesaurus. Code C8858.

Follicular lymphoma grade I, II, III that is resistant to treatment. 